Annales Geophysicae, 23, 3365-3373, 2005

SRef-ID: 1432-0576/ag/2005-23-3365

(C) European Geosciences Union 2005

\title{
Energy release and conversion by reconnection in the magnetotail
}

\author{
J. Birn ${ }^{1}$ and M. Hesse ${ }^{2}$ \\ ${ }^{1}$ Los Alamos National Laboratory, Los Alamos, New Mexico, USA \\ ${ }^{2}$ NASA Goddard Space Flight Center, Greenbelt, Maryland, USA
}

Received: 14 June 2005 - Revised: 31 August 2005 - Accepted: 16 September 2005 - Published: 30 November 2005

\begin{abstract}
Magnetic reconnection is the crucial process in the release of magnetic energy previously stored in the magnetotail in association with substorms. However, energy transfer and dissipation in the vicinity of the reconnection site is only a minor part of the energy conversion. We discuss the energy release, transport, and conversion based on largescale resistive MHD simulations of magnetotail dynamics and more localized full particle simulations of reconnection. We address in particular, where the energy is released, how it propagates and where and how it is converted from one form into another. We find that Joule (or ohmic) dissipation plays only a minor role in the overall energy transfer. Bulk kinetic energy, although locally significant in the outflow from the reconnection site, plays a more important role as mediator or catalyst in the transfer between magnetic and thermal energy. Generator regions with potential auroral consequences are located primarily off the equatorial plane in the boundary regions of the plasma sheet.
\end{abstract}

Keywords. Magnetospheric physics (magnetospheric configuration and dynamics; storms and substorms) - Space plasma physics (magnetic reconnection)

\section{Introduction}

The release of previously stored magnetic energy and its conversion into particle energy in the form of heating, bulk plasma kinetic energy, and accelerated particles with high nonthermal energies is perhaps the most important aspect of magnetic reconnection. Here we investigate details of the energy release and conversion process in the framework of simulations of magnetotail reconnection, relevant for magnetospheric substorms and perhaps also more localized energy releases ("pseudo-onsets"). The present investigations are based largely on MHD simulations using an (ad hoc) re-

Correspondence to: J. Birn

(jbirn@lanl.gov) sistivity to model the necessary nonideal contribution to the electric field, which provides dissipation and enables reconnection. In more realistic, collisionless, models of reconnection in the magnetotail, the dissipation results primarily from electron inertia which causes nongyrotropy of the electron pressure tensor (e.g., Vasyliūnas, 1975; Hesse et al., 1999, 2001). However, the fact that the dissipation is highly localized in our simulations and contributes only negligibly to the overall energy conversion suggests that the MHD results may be relevant more generally even if the dissipation is not governed by resistivity. A comparison of the MHD results with the dissipation in a (more localized) particle simulation of reconnection supports this view. We note that quantitative results on energization and energy conversion depend strongly on the system size considered. In smaller systems, such as those usually considered in particle simulations of magnetic reconnection, the actual dissipation mechanism and its relative importance within the overall energy conversion are more substantial than in a large-scale configuration.

We start our investigations in Sect. 2 by considering the individual equations that govern the conservation of various forms of energy and the transfer from one form into another. In Sect. 3 we then investigate the relevant terms on the basis of MHD and (to a minor extent) particle-in-cell simulations of reconnection. In Sect. 4 we will focus particularly on the generator regions in the tail and the contributing mechanisms.

\section{Energy equations}

The energy transport and conversion are governed by conservation laws that can be derived as moments of the Vlasov equation governing the particle distribution in phase space, in combination with Maxwell's equations (e.g., Akhiezer et al., 1975). In this section we consider the individual equations that govern separate forms of energy and the transfer from one form to another, which are then to be analyzed in the 
subsequent section. We start with the energy of the magnetic field governed by Faraday's law

$\frac{\partial \boldsymbol{B}}{\partial t}=-\nabla \times \boldsymbol{E}$

Taking the scalar product of Eq. (1) with $\boldsymbol{B}$ and using Ampère's law

$\nabla \times \boldsymbol{B}=\mu_{0} \boldsymbol{j}$

one finds "Poynting's theorem", governing magnetic energy transport:

$\frac{\partial}{\partial t} \frac{B^{2}}{2 \mu_{0}}=-\nabla \cdot\left(\frac{\boldsymbol{E} \times \boldsymbol{B}}{\mu_{0}}\right)-\boldsymbol{j} \cdot \boldsymbol{E}$

Here we use MKS units and standard notations with $\boldsymbol{E}$ denoting the electric field, $\boldsymbol{B}$ the magnetic induction, $\boldsymbol{j}$ electric current density and $\mu_{0}$ the permeability of free space. Electromagnetic energy flow is contained in the Poynting flux vector $\boldsymbol{S}=\boldsymbol{E} \times \boldsymbol{B} / \mu_{0}$. On the left-hand side of Eq. (3), the electric field energy density, $\epsilon_{0} E^{2} / 2$, does not appear, consistent with our neglect of the displacement current in Ampere's law, Eq. (2), and the assumption of quasi-neutrality, valid for transport and wave speeds well below the speed of light.

Equation (3) can be further rewritten using the electric field in the plasma rest frame, $\boldsymbol{E}^{\prime}=\boldsymbol{E}+\boldsymbol{v} \times \boldsymbol{B}$,

$\frac{\partial}{\partial t} \frac{B^{2}}{2 \mu_{0}}=-\nabla \cdot\left(\frac{\boldsymbol{E} \times \boldsymbol{B}}{\mu_{0}}\right)-\boldsymbol{v} \cdot(\boldsymbol{j} \times \boldsymbol{B})-\boldsymbol{j} \cdot \boldsymbol{E}^{\prime}$

The electric field $\boldsymbol{E}^{\prime}$ is governed by the generalized Ohm's law (e.g., Vasyliūnas, 1975)

$\boldsymbol{E}+\boldsymbol{v} \times \boldsymbol{B}=\eta \boldsymbol{j}+\frac{1}{n e}\left(\boldsymbol{j} \times \boldsymbol{B}-\nabla \cdot \mathbf{P}_{\mathbf{e}}\right)+\frac{m_{e}}{n e^{2}}\left[\frac{\partial \boldsymbol{j}}{\partial t}+\nabla \cdot(\boldsymbol{j} \boldsymbol{v}+\boldsymbol{v} \boldsymbol{j})\right]$

where $n$ is the plasma density, $m_{e}$ the electron mass, $\eta$ the resistivity, and $\mathbf{P}_{\mathbf{e}}$ denotes the electron pressure tensor, evaluated in the plasma rest frame. This electric field is responsible for the dissipation associated with reconnection. In the kinetic regime, as investigated by particle simulations, $\boldsymbol{E}^{\prime}$ usually is dominated by the electron pressure tensor term in Eq. (5) (Vasyliūnas, 1975; Lyons and Pridmore-Brown, 1990; Hesse and Winske, 1993). Note that the Hall term, $\boldsymbol{j} \times \boldsymbol{B} / n e$, does not contribute to $\boldsymbol{j} \cdot \boldsymbol{E}^{\prime}$. In resistive MHD simulations the dissipation term is represented by ohmic heating, $\eta j^{2}$.

When heat flux (defined as thermal energy flux in the plasma rest frame) is neglected, the thermal and kinetic energy transport is governed by (Akhiezer et al., 1975)

$\frac{\partial}{\partial t}\left(u+\frac{\rho}{2} v^{2}\right)=-\nabla \cdot\left[\left(u+p+\frac{\rho}{2} v^{2}\right) \boldsymbol{v}\right]+\boldsymbol{j} \cdot \boldsymbol{E}$

Here, $\rho \approx n m_{i}$ is the mass density, $u=3 p / 2$ is the thermal energy density of the plasma, and isotropic pressure is assumed. Equations (3) and (6) can be added together to yield the conservation law of total energy

$$
\frac{\partial}{\partial t}\left(\frac{B^{2}}{2 \mu_{0}}+u+\frac{\rho}{2} v^{2}\right)=-\nabla \cdot\left[\frac{\boldsymbol{E} \times \boldsymbol{B}}{\mu_{0}}+(u+p) \boldsymbol{v}+\frac{\rho}{2} v^{2} \boldsymbol{v}\right] \text { (7) }
$$

where the total energy flux on the right-hand side is the sum of Poynting flux, $\boldsymbol{S}=\boldsymbol{E} \times \boldsymbol{B} / \mu_{0}$, enthalpy flux, $\boldsymbol{H}=(u+p) \boldsymbol{v}$, and bulk kinetic energy flux, $\boldsymbol{K}=\rho v^{2} \boldsymbol{v} / 2$.

Equation (6) governs the sum of thermal and bulk flow kinetic energy. It is instructive, however, to derive separate equations for the two energy terms. The transport equation for the kinetic energy of the plasma bulk flow follows from taking the scalar product of the velocity vector $\boldsymbol{v}$ with the momentum equation

$\rho \frac{\mathrm{d} \boldsymbol{v}}{\mathrm{d} t}=\rho\left(\frac{\partial \boldsymbol{v}}{\partial t}+\boldsymbol{v} \cdot \nabla \boldsymbol{v}\right)=\boldsymbol{j} \times \boldsymbol{B}-\nabla p$

and using the continuity equation (mass conservation) given by

$$
\frac{\partial \rho}{\partial t}=-\nabla \cdot \rho \boldsymbol{v}
$$

This yields

$$
\frac{\partial}{\partial t} \frac{\rho}{2} v^{2}=-\nabla \cdot\left(\frac{\rho}{2} v^{2} \boldsymbol{v}\right)+\boldsymbol{v} \cdot(\boldsymbol{j} \times \boldsymbol{B}-\nabla p)
$$

If Eq. (10) is subtracted from Eq. (6) we obtain an equation for the thermal energy transport

$\frac{\partial u}{\partial t}=-\nabla \cdot[(u+p) \boldsymbol{v}]+\boldsymbol{v} \cdot \nabla p+\boldsymbol{j} \cdot \boldsymbol{E}^{\prime}$

where $\boldsymbol{E}^{\prime}$ again is the electric field in the plasma rest frame, given by the right-hand side of Eq. (5). The first two terms on the right-hand side of Eq. (11) describe adiabatic, i.e., entropy conserving, compression or expansion. The Joule dissipation term $\boldsymbol{j} \cdot \boldsymbol{E}^{\prime}$ (ohmic dissipation in resistive MHD) provides the direct transfer from magnetic to thermal energy.

The left-hand sides of Eqs. (4), (10) and (11), together with the first terms on the right-hand sides, represent the conservation of each of the different forms of energy, while the remaining terms represent the conversion (transfer) of one form of energy into another. In Eq. (3), $\boldsymbol{j} \cdot \boldsymbol{E}$ governs the conversion between magnetic and kinetic (thermal and bulk flow) energy. Positive $\boldsymbol{j} \cdot \boldsymbol{E}$ corresponds to a load; negative $\boldsymbol{j} \cdot \boldsymbol{E}$ corresponds to a generator. Since Joule dissipation typically is positive (ohmic dissipation always is positive), a generator requires plasma motion against the Lorentz force. As demonstrated by Eq. (10), the associated energy can be supplied either by kinetic bulk flow energy or by mechanical work, $-\boldsymbol{v} \cdot \nabla p$, that derives from thermal energy or compression via Eq. (11). It should be noted that the generator term also is not altered if the Hall electric field is included from Eq. (5). However, it depends on the frame in which it is evaluated.

As mentioned above, one part of the $\boldsymbol{j} \cdot \boldsymbol{E}$ term, Joule (or ohmic) heating, goes directly into thermal energy (Eq. 11), while the remaining part represents the acceleration (or deceleration) by Lorentz forces, which affects kinetic bulk flow energy (Eq. 10). The combination of Eqs. (10) and (11) shows that, in approximate force balance, this kinetic energy also is transferred into thermal energy via mechanical work by the pressure gradient force. Equations (4), (10) and (11) 
also illustrate that the transfer of internal (i.e., thermal) energy to magnetic energy requires as an intermediate step the conversion to kinetic bulk flow energy.

We note that the conservation laws discussed above also apply to the kinetic regime, as treated in particle simulations, however, with some modifications. We already discussed that Joule dissipation in the immediate vicinity of the reconnection site predominantly results from the divergence of the nongyrotropic part of the electron pressure tensor (Hesse et al., 1999, 2001). Furthermore, the full pressure tensor, dominated by the ions, need not be isotropic. Indeed such anisotropy is found to govern the structure in the surrounding region where Hall electric fields are important (e.g., Yin et al., 2002). Finally, heat flux may also play a role (Hesse et al., 2004).

In the following sections we will discuss the various contributions to the energy equations on the basis of a simulation of magnetotail dynamics associated with reconnection in the near tail (Birn and Hesse, 1996), which has also been the basis for previous investigations of current disruption and diversion (Birn et al., 1999). The simulation covers a tail region from $x=-5 R_{E}$ to $x=-65 R_{E}$ with a dipole located outside the simulation box at $x=0$. The field evolution of this simulation is characterized by the onset of magnetic reconnection at $t \approx 2$ min near $x=-23 R_{E}$, triggered by imposing finite resistivity for $t \geq 0$ after a period of thinning and current intensification in the near tail. This leads to plasmoid formation and ejection into the far tail and to a field collapse (dipolarization) of the inner tail. Here we consider a particular snapshot of the evolution when magnetic reconnection has advanced and the plasmoid has moved downtail, typical for the main phase of the evolution, which may be associated with the substorm expansion phase. In Sect. 3 we will focus on the standard view in the noon-midnight meridianal plane, also including a comparison with local dissipation results obtained in a particle simulation of reconnection (Hesse et al., 1999). In Sect. 4 we will discuss the identification of load and generator regions in an equatorial projection and in a characteristic cross-section of the near tail.

In the following we will use dimensionless quantities, normalized by a typical magnetic field strength $B_{n}$ (the lobe field at the near-Earth boundary), the plasma sheet density $\rho_{n}$ at the near-Earth boundary, and a scale length $L_{n}$, representing the plasma sheet half-width at the near-Earth boundary. This leads to a typical Alfvén speed $v_{n}=B_{n} / \sqrt{\mu_{o} \rho_{n}}$ and an electric field $E_{n}=v_{n} B_{n}$. For illustration, we choose $B_{n}=40 n T$, $L_{n}=6000-12000 \mathrm{~km} \approx 1-2 R_{E}$, and $v_{n}=1000 \mathrm{~km} / \mathrm{s}$, leading to $t_{n}=6-12 \mathrm{~s}$. For simplicity and easier understanding, however, we will use the same symbols as for the dimensional quantitities.

\section{Energy release and conversion}

Figure 1 provides an overview of the configuration at $t=80$ (corresponding to 8 to $16 \mathrm{~min}$ after turning on resistivity), showing magnetic field lines (solid contours), the cross-tail

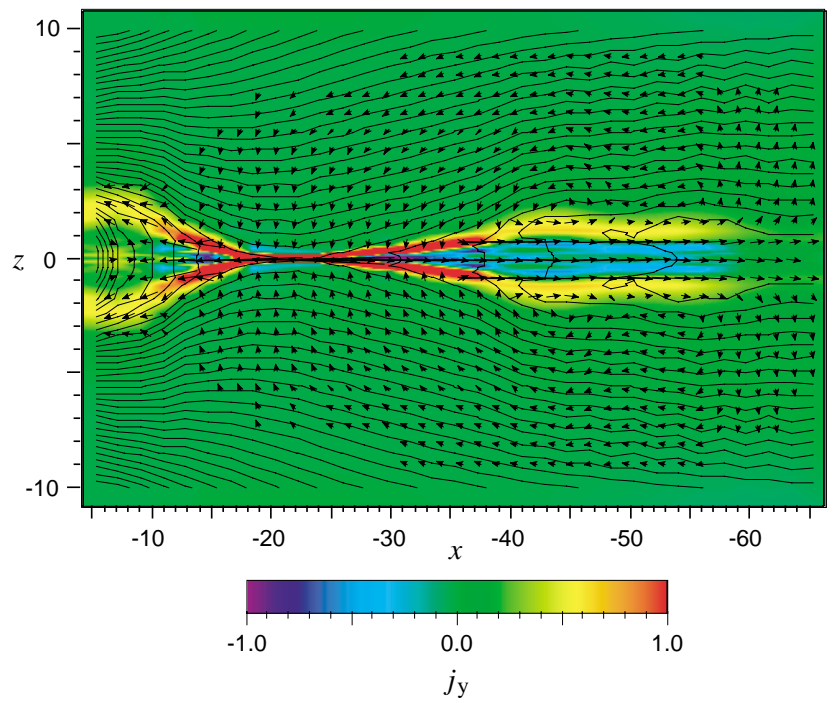

Fig. 1. Magnetic field lines, cross-tail current density (color), and energy flow vectors (arrows) in the midnight meridianal plane, $y=0$, at $t=80$, based on a simulation of magnetotail dynamics (Birn and Hesse, 1996).

current density (color), and the total energy flux vectors (the sum of the flux vectors on the RHS of Eq. 7) in the midnight meridianal plane, $y=0$. It demonstrates the expected energy flow from the lobe regions through current layers that might be identified as slow shocks, into the inner plasma sheet earthward and tailward of the reconnection site. The detailed magnetic field structure in the vicinity of the current layers tailward from the reconnection site shows a reversal of the main field component $B_{x}$. This structure is not consistent with a pure slow shock; it requires the additional presence of an Alfvén wave (Vasyliūnas, 1975).

For a full understanding of the energy release, transport, and conversion, one needs to consider all terms in Eqs. (4), (10) and (11). We start with an overview of the different forms of energy fluxes (Fig. 2), showing Poynting flux, $\boldsymbol{S}=\boldsymbol{E} \times \boldsymbol{B}$, enthalpy flux, $\boldsymbol{H}=(u+p) \boldsymbol{v}$, and kinetic energy flux, $\boldsymbol{K}=\left(\rho v^{2} / 2\right) \boldsymbol{v}$, respectively, at $t=80$, integrated over $y$ from $y=0$ to $y=10$, together with the magnetic field lines in the midnight meridianal plane $(y=0)$. Figure 2 demonstrates that the character of the energy transport changes across the slow shocks from, predominantly, Poynting flux to, predominantly, enthalpy flux and, to a minor extent, bulk kinetic energy flux.

Figure 3 shows the changes of the three forms of energy density at $t=80$, given by the left-hand sides of Eqs. (4), (10) and (11), integrated over $y$, again together with the magnetic field lines in the midnight meridianal plane $(y=0)$. (A local picture at $y=0$ would look very similar.) Figure 4 shows the divergence of the energy fluxes, given by the first term on each of the right-hand sides of Eqs. (4), (10) and (11), respectively, also integrated over $y$. Finally, Fig. 5 shows the conversion of energy from one form to another, given by the transfer terms on the right-hand sides of Eqs. (4), (10) and 


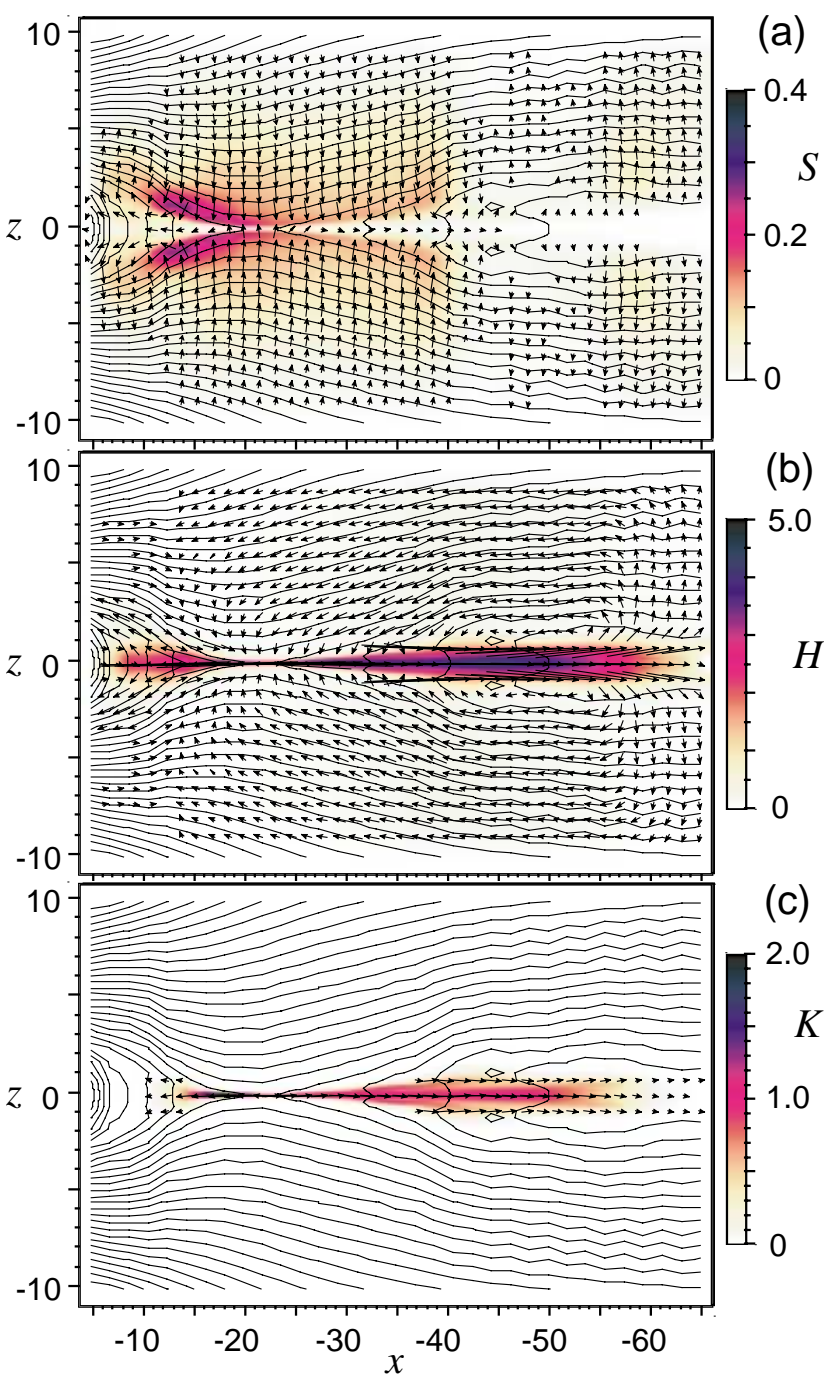

Fig. 2. Energy fluxes at $t=80$, integrated over $y$ from $y=0$ to $y=10$ : (a) Poynting flux, $\boldsymbol{S}=\boldsymbol{E} \times \boldsymbol{B}$, (b) enthalpy flux, $\boldsymbol{H}=(p+u) \boldsymbol{v}$, and (c) kinetic energy flux, $\boldsymbol{K}=\left(\rho v^{2} / 2\right) \boldsymbol{v}$. Color indicates the magnitude of the fluxes, while the arrows show the flux vectors. Only flux vectors with fluxes exceeding 0.005 in magnitude are shown.

(11), again integrated over $y$. We note that Figs. 3, 4, and 5 all use the same color scale, shown in Figs. 3 and 4.

We will now discuss the changes, transport, and conversion of energy, following the direction of the energy transport from the lobes, through the slow shocks, to the near and far plasma sheet. Figure 3a shows the expected release of magnetic energy from the lobes, which covers a wide region from $x \approx-10$ to $x \approx-40$. As demonstrated by Fig. $4 \mathrm{a}$, the reduction in magnetic energy corresponds to a divergence of Poynting flux covering the same region. This leads to the Poynting flux shown in Fig. 2a.

The next region to consider are the slow shocks, also including the reconnection site. In this region, Poynting flux is lost (Fig. 4a) and converted through a positive load term $\boldsymbol{j} \cdot \boldsymbol{E}$ (Fig. 5a; Eq. 4) into kinetic energy flux (Fig. 4c;

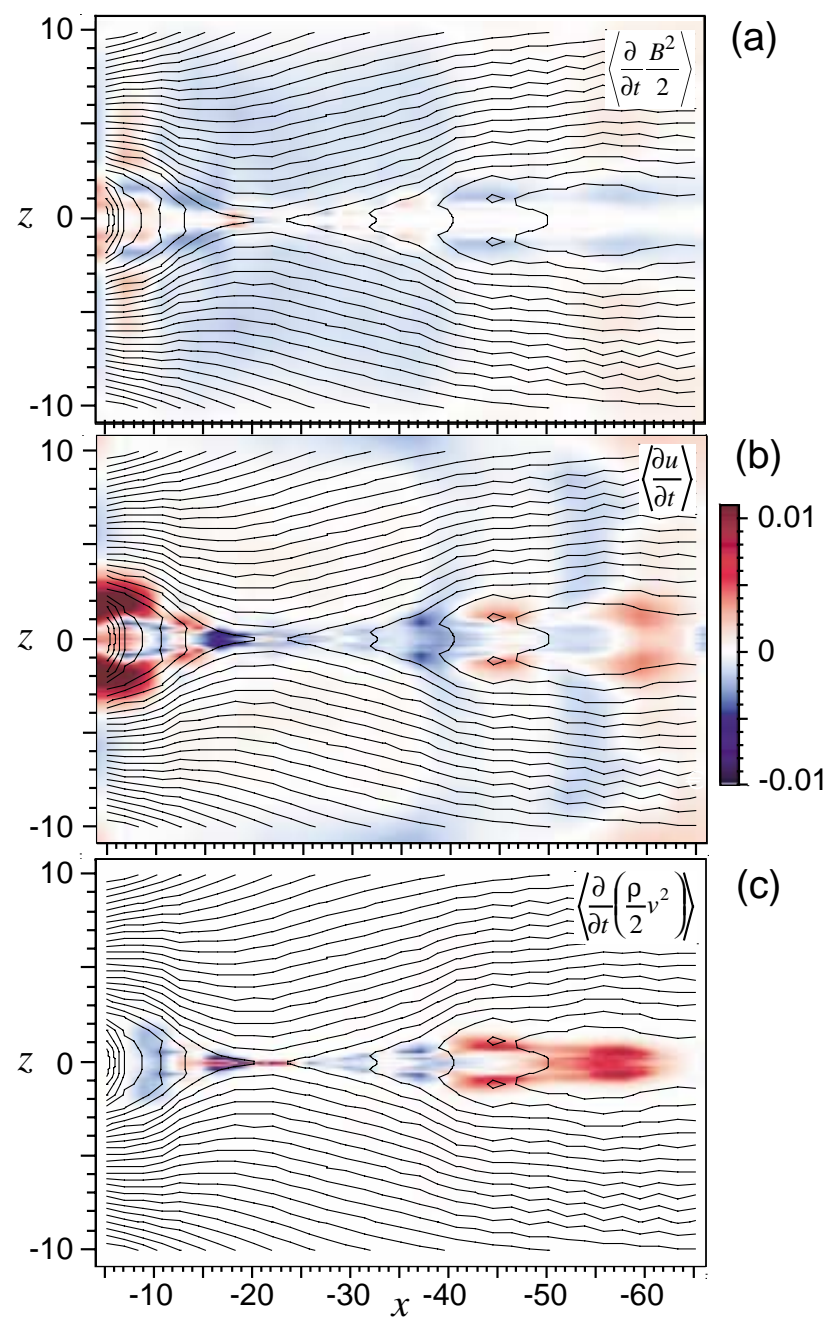

Fig. 3. Change of energy densities, integrated over $y$ : (a) magnetic energy, (b) thermal energy, and (c) kinetic energy density.

Eq. 10). However, through approximate force balance, most of this kinetic energy is immediately transferred to enthalpy flux (Figs. 4b, 5c; Eqs. 10 and 11), such that the enthalpy flux is the dominant flux exiting from the slow shock regions (Fig. 2). Only a small amount of the loss in Poynting flux goes directly into heating, via Joule dissipation (Fig. 5b; Eq. 11).

We now follow the energy flow further into the inner part of the near tail, where there is obvious braking of the flow (Fig. 5c), which causes loss of kinetic energy flux (Fig. 4c). Most of this energy is transferred, via mechanical energy $(v \cdot \nabla p$, Fig. 5d), to enthalpy flux (Fig. 4b, Eq. 11), while it causes no significant heating (Fig. 3b). However, even closer in, but at higher $|z|$ values, we find significant compression, which leads to a loss of enthalpy flux (Fig. 4b) and heating (increase in thermal energy; Fig. 3b).

In the MHD simulation, we find overall very little Joule dissipation (Fig. 5b), concentrated near the reconnection site and the adjacent slow shocks. This dissipation result is based on the most artificial term in the energy balance, the ad hoc 


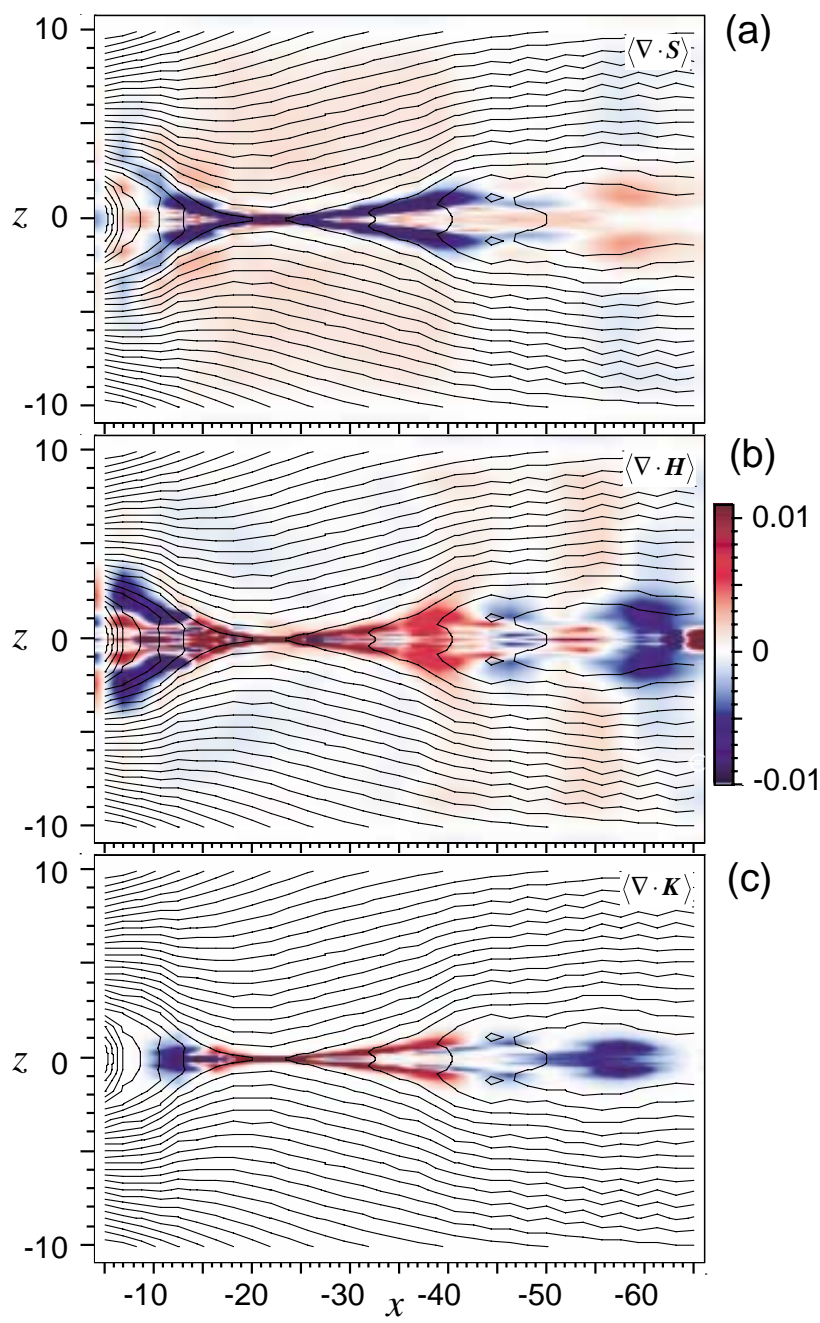

Fig. 4. Divergence of the energy fluxes, shown in Fig. 2, integrated over $y$ : (a) Poynting flux, (b) enthalpy flux, (c) kinetic energy flux.

resistivity, which in the simulation was assumed uniform. Therefore we compare this ohmic dissipation with results from a PIC simulation, which focuses on the vicinity of the reconnection site. Figure 6 shows the magnetic field and current density (top), the load regions $(\boldsymbol{j} \cdot \boldsymbol{E}>0$ ) (center), and the Joule dissipation $\left(\boldsymbol{j} \cdot \boldsymbol{E}^{\prime}\right)$ based on a two-dimensional simulation of magnetic reconnection (Hesse et al., 1999). As in the MHD simulation, the load regions and the Joule dissipation are localized in the vicinity of the reconnection site and in thin current layers extending out from the reconnection site. The most noticable difference is the absence of the slow shocks in the particle simulation; the equivalent load regions in the PIC simulation extend along the separatrices. There are also additional load regions associated with the current concentration in the highly curved fields of the magnetic islands. In contrast to the load regions, the Joule dissipation $\left(\boldsymbol{j} \cdot \boldsymbol{E}^{\prime}\right)$ occurs mostly at the central reconnection site, similar to the resistive MHD case (Fig. 5b).

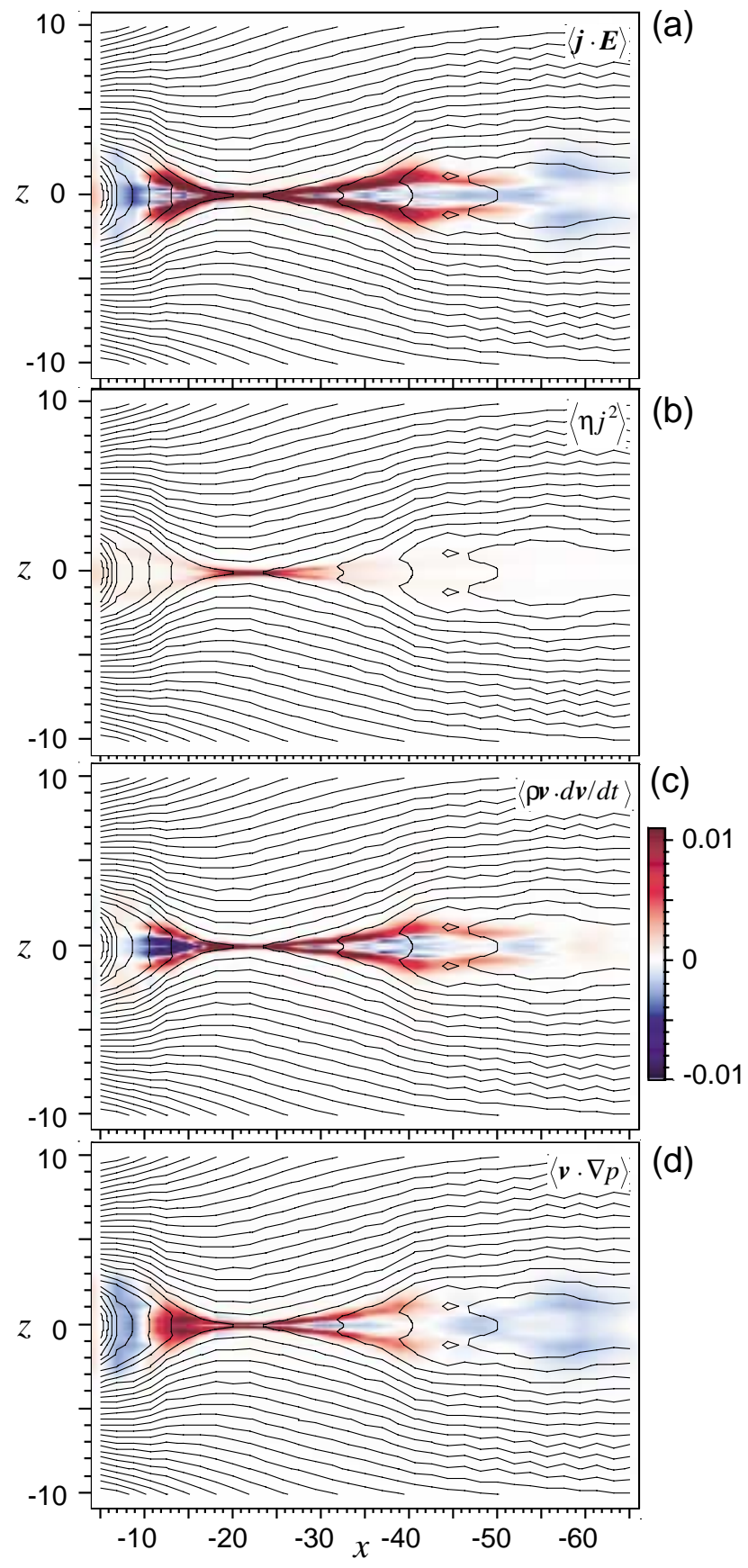

Fig. 5. Energy transfer terms, integrated over $y$ : (a) $\boldsymbol{j} \cdot \boldsymbol{E}$, (b) ohmic heating $\eta j^{2}$, (c) kinetic energy transfer $\rho v \cdot d v / d t$, (d) transfer between kinetic and thermal energy $v \cdot \nabla p$.

\section{Generator regions}

We now investigate properties of generator and load mechanisms in an equatorial view, focusing aggain on the MHD simulation. We investigate particularly the relative importance of the contributions to the energy transfer term $\boldsymbol{j} \cdot \boldsymbol{E}$. The results are shown in Figs. 7 and 8 . Ohmic dissipation, $\eta j^{2}$, is highly localized and so small that it would not show in the color representation of Fig. 7. Hence 
(a)

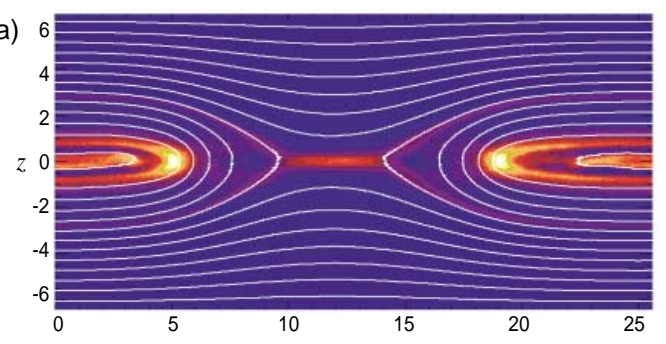

(b)
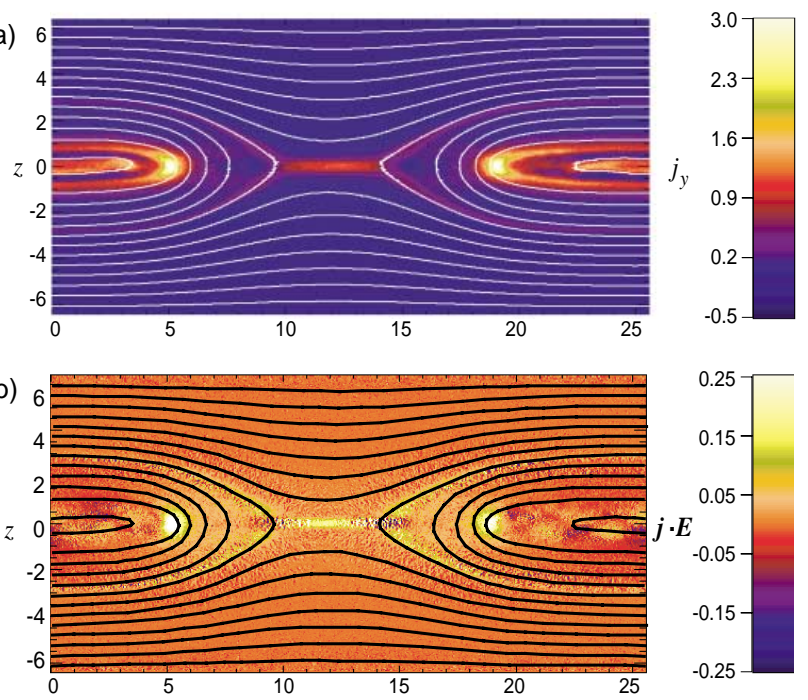

(c)

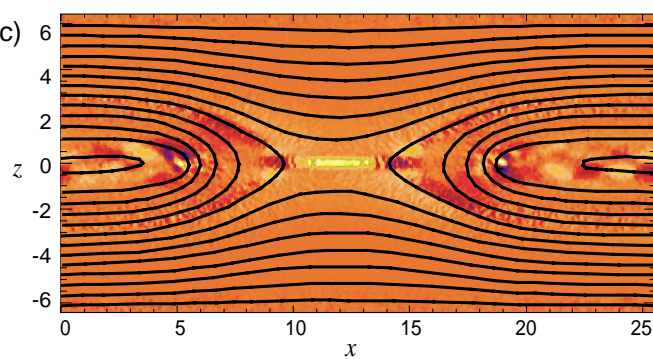

Fig. 6. Particle-in-cell simulations of magnetic reconnection, showing (a) current density and magnetic field, (b) load regions, defined by $\boldsymbol{j} \cdot \boldsymbol{E}>0$, and (c) Joule dissipation, given by $\boldsymbol{j} \cdot \boldsymbol{E}^{\prime}$.

$$
\begin{aligned}
\boldsymbol{j} \cdot \boldsymbol{E} & \approx-\boldsymbol{j} \cdot(\boldsymbol{v} \times \boldsymbol{B})=\boldsymbol{v} \cdot(\boldsymbol{j} \times \boldsymbol{B}) \\
& =\boldsymbol{v} \cdot \nabla p+\boldsymbol{v} \cdot \rho \mathrm{d} \boldsymbol{v} / \mathrm{d} t
\end{aligned}
$$

Figure 7 shows the color-coded magnitude of $\boldsymbol{j} \cdot \boldsymbol{E}$ (top), the pressure gradient term (center), and the inertial contributions to $\boldsymbol{j} \cdot \boldsymbol{E}$ (bottom), all integrated over $z$ from $z=0$ to $z=10$, together with flow velocity vectors and the magnetic neutral line in the equatorial plane. Load regions $(\boldsymbol{j} \cdot \boldsymbol{E}>0)$ are indicated by yellow and red and generator regions $(\boldsymbol{j} \cdot \boldsymbol{E}<0)$ by blue or purple color. (We note again that the value of $\boldsymbol{j} \cdot \boldsymbol{E}$, and hence the interpretation of generator and load, are frame dependent and have to be distinguished from ohmic, or more generally, Joule dissipation, $\boldsymbol{j} \cdot \boldsymbol{E}^{\prime}$, where $\boldsymbol{E}^{\prime}$ is the electric field in the plasma rest frame.)

Figure 7a shows that the reconnection site, as well as the regions earthward and tailward of it, act as loads. Generator regions are present towards the flanks, where the flow is diverted away from midnight. This is also the region where the flow diversion distorts the magnetic field, leading to the build-up of field-aligned currents (Plate 1 of Birn et al., 1999). The generator regions hence roughly coincide with the regions where part of the cross-tail current gets diverted to the field-aligned currents of the substorm current wedge. This may not be too surprising, because both the evaluation

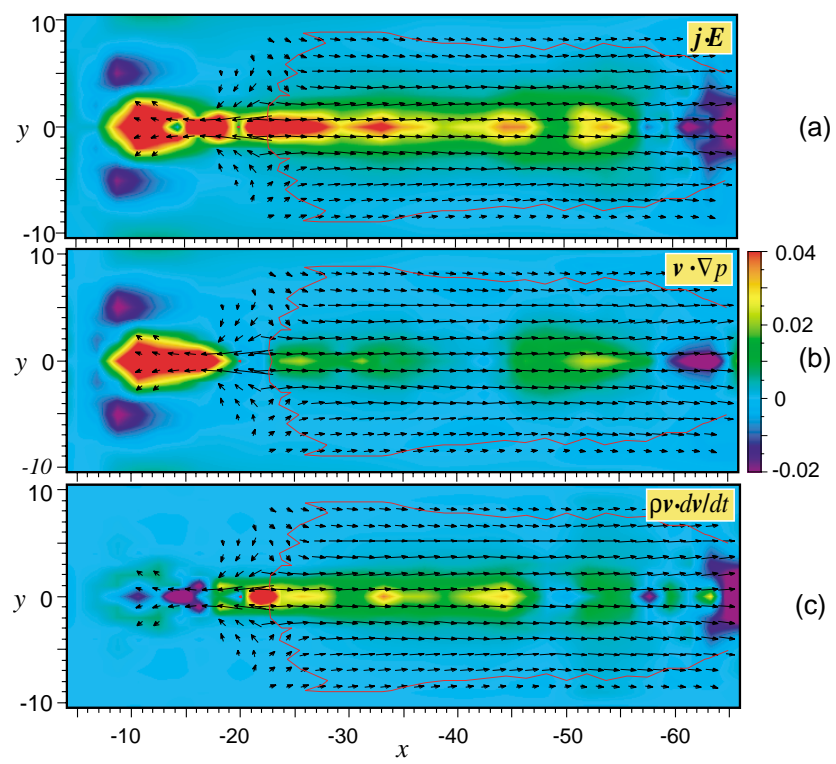

Fig. 7. Generator and load regions, integrated over $z$ from $z=0$ to $z=10$, and projected into the equatorial plane: (a) $\boldsymbol{j} \cdot \boldsymbol{E}$, (b) pressure gradient contribution $\boldsymbol{v} \cdot \nabla p,(\mathbf{c})$ inertial contribution $\rho \boldsymbol{v} \cdot d \boldsymbol{v} / d t$. Red color indicates load regions, blue corresponds to generator regions. The red contour represents the magnetic neutral line.

of generator regions and current diversion rely on the force balance, Eq. (8). We note, however, that the generator evaluation takes only the components of the forces in the direction of the flow into account.

The dominant contribution to $\boldsymbol{j} \cdot \boldsymbol{E}$ stems from the pressure gradient term in Eq. (12). This is illustrated by Figs. 7b and $\mathrm{c}$, showing the pressure gradient and the inertial contribution, respectively, as function of $x$ and $y$, again integrated over $z$. The inertia term provides a generator mechanism in the region of flow braking, as postulated by Shiokawa et al. (1997). In the integral evaluation, however, this term is overcompensated by a load term from the pressure contribution, located at higher $|z|$ (Fig. 5a).

Details of the generation regions and mechanisms in the near tail at $|y| \approx 5$ are demonstrated by Fig. 8 for a tail crosssection at $x=-8.75$, showing (a) $\boldsymbol{j} \cdot \boldsymbol{E}$, (b) $\nabla \cdot \boldsymbol{S}$, (c) $\boldsymbol{v} \cdot \nabla p$, (d) $\nabla \cdot \boldsymbol{H}$ where $\boldsymbol{H}=(u+p) \boldsymbol{v}$ is the enthalpy flux, (e) the crosstail current density $j_{y}$, and (f) the earthward flow velocity component $v_{x}$. Note that the color scale in Fig. 8d differs from those in Figs. 8a-c. Solid lines represent the boundaries of the closed field line region (separatrices), and arrows show the projection of velocity vectors perpendicular to the magnetic field. Figure 8 a demonstrates that the generator regions (blue) near $|y|=5$ are located not in the equatorial plane but away from it; this is consistent with the current diversion found earlier (Birn and Hesse, 1996; Birn et al., 1999). The generator regions are part of a vortical flow pattern, which is also associated with the build-up of the field-aligned currents of the substorm current wedge. The energy stems from mechanical work that is done by flow toward the lobes in the direction of the pressure gradient force $(v \cdot \nabla p<0$; Fig. 8c). 

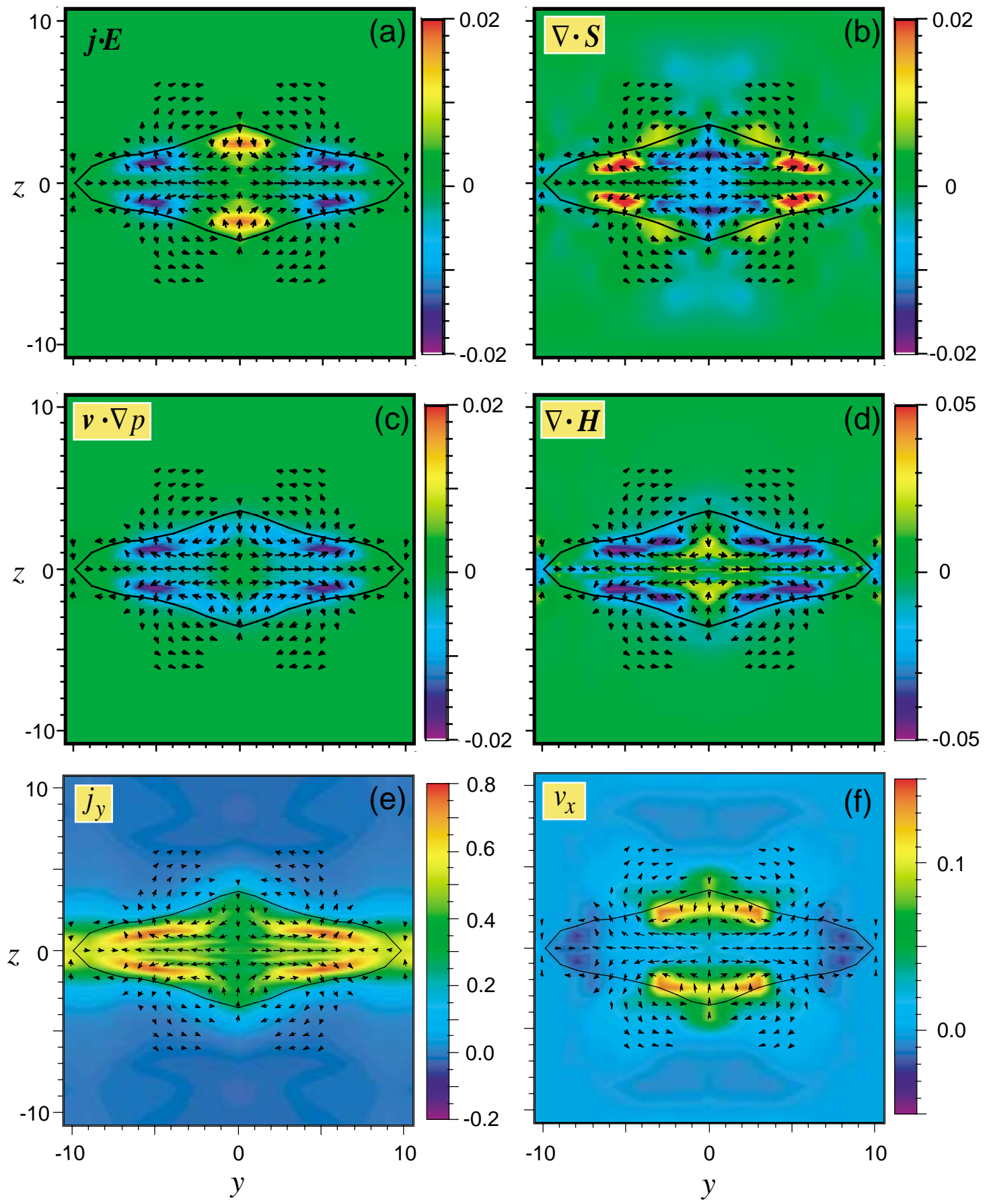

Fig. 8. Characteristic properties near the generator and load regions at $x=-8.75$ for $t=100$ : (a) $\boldsymbol{j} \cdot \boldsymbol{E}$, (b) $\nabla \cdot \boldsymbol{S}$, (c) $\boldsymbol{v} \cdot \nabla p,(\mathbf{d}) \nabla \cdot \boldsymbol{H}$ where $\boldsymbol{H}=(u+p) \boldsymbol{v},(\mathbf{e})$ cross-tail current density $j_{y}$, and (f) earthward velocity $v_{x}$. Note that the color scale in (d) differs from (a-c). Solid lines represent the boundaries of the closed field line region (separatrices) and arrows show the projection of velocity vectors perpendicular to the magnetic field. Panel (a) indicates generator regions (blue) near $|y|=5$, where positive (earthward) Poynting flux is generated ( $\nabla \cdot S>0$, panel b). The generation mechanism involves a loss of enthalpy flux $(\nabla \cdot \boldsymbol{H}<0$, panel d) and mechanical work $(\boldsymbol{v} \cdot \nabla p<0$, panel c). The generator regions are also closely associated with bifurcated current layers (panel e), just outside of the earthward flow regions (panel f).

This energy is converted to Poynting flux $(\nabla \cdot S>0$; Fig. 8 b), consistent with an approximate balance of the two terms on the right-hand side of Poynting's theorem (Eq. 3). Figure 8d demonstrates that the energy that goes into the Poynting flux actually stems from a loss of enthalpy flux. Part of this ther- mal energy goes into local heating (Fig. 3b), while the rest is transferred to mechanical work. As shown above, through approximate force balance, this energy is then immediately transferred to magnetic energy and Poynting flux, rather than staying as kinetic energy. 


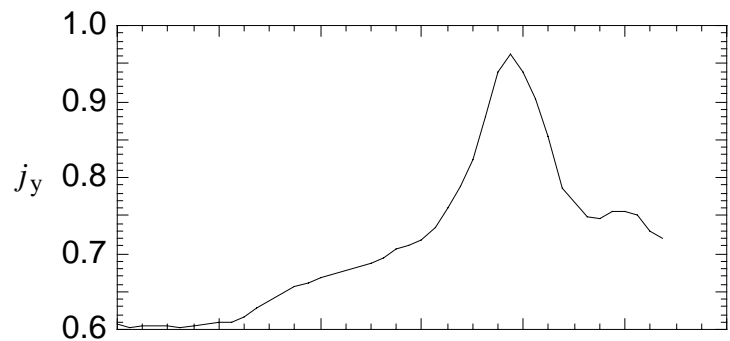

(a)
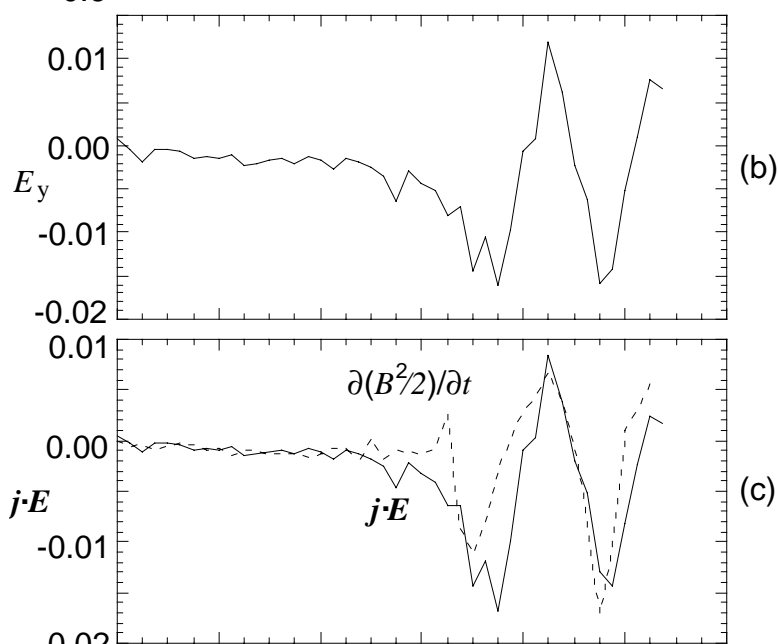

(c)

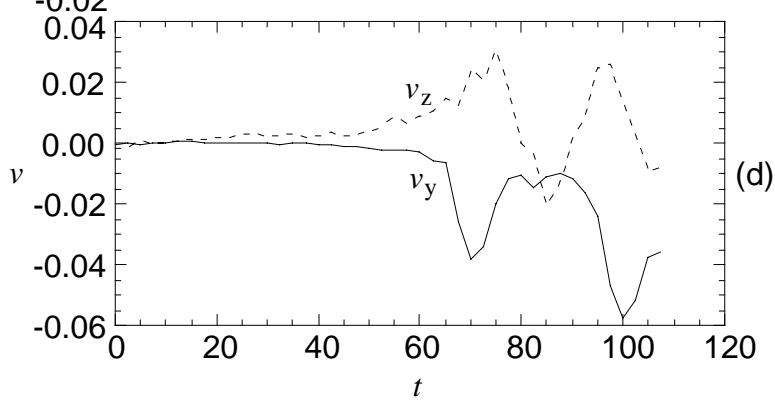

Fig. 9. Characteristic quantities in the generator region at $x=-8.75, y=-4, z=1.2$ as functions of time, (a) cross-tail current density, (b) cross-tail electric field, (c) $\boldsymbol{j} \cdot \boldsymbol{E}$ (solid line) and the local derivative of the magnetic energy density, $\partial\left(B^{2} / 2\right) / \partial t$ (dashed line), and (d) the velocity components $v_{y}$ (solid line) and $v_{z}$ (dashed line).

Figures $8 \mathrm{e}-\mathrm{f}$ further demonstrate that the generator regions are associated with bifurcated currents that peak in sheets away from the equatorial plane and that they are located near the edges of (moderately) fast earthward flow regions in the plasma sheet boundary layers. Both of these features, as well as the association with flow components toward the lobes, appear consistent with recent identifications of generator regions from Cluster data (Marghitu et al., 2005 ${ }^{1}$; Hamrin et al., $2005^{2}$ ).

\footnotetext{
${ }^{1}$ Marghitu, O., Hamrin, M., Klecker, B., Vaivads, A., McFadden, J., Buchert, S., Kistler, L. M., Dandouras, I., André, M., and Rème, H.: Experimental investigation of auroral generator regions with conjugated Cluster and FAST data, Ann. Geophys., submitted, 2005.

${ }^{2}$ Hamrin, M., Marghitu, O., Rönnmark, K., Klecker, B., André, M., Buchert, S., Kistler, L. M., McFadden, J., Rème, H., and
}

The generator mechanism in the boundary regions of the plasma sheet, shown in Fig. 8, is not very steady. This is demonstrated by Fig. 9, which shows several characteristic quantities in the generator region as functions of time, (a) cross-tail current density, (b) cross-tail electric field, (c) $\boldsymbol{j} \cdot \boldsymbol{E}$ (solid line) and the local derivative of the magnetic energy density, $\partial\left(B^{2} / 2 / \partial t\right.$ (dashed line), and (d) the velocity components $v_{y}$ (solid line) and $v_{z}$ (dashed line). Negative intensifications of $\boldsymbol{j} \cdot \boldsymbol{E}$ occur particularly at $t=75$ and $t=95$. They are associated with negative cross-tail electric fields corresponding to upward (positive $z$ ) and outward (negative $y$ ) plasma motion. For comparison with the observations by Marghitu et al. (2005) ${ }^{1}$ and Hamrin et al. (2005) ${ }^{2}$ it is instructive to apply dimensional units to our results, based on a magnetic field unit of $40 \mathrm{nT}$, a velocity unit (Alfvén speed) of $1000 \mathrm{~km} / \mathrm{s}$, and a length unit of $12000 \mathrm{~km} \approx 2 R_{E}$. This leads to a current density unit of $2.7 \mathrm{nA} / \mathrm{m}^{2}$, an electric field unit of $40 \mathrm{mV} / \mathrm{m}$, and a unit of $\sim 10^{-10} \mathrm{~W} / \mathrm{m}^{3}$ for $\boldsymbol{j} \cdot \boldsymbol{E}$ and $\partial\left(B^{2} / 2\right) / \partial t$. Thus we find a current intensification of a few $\mathrm{nA} / \mathrm{m}^{2}$, negative electric field spikes of $\sim 0.8 \mathrm{mV} / \mathrm{m}$, separated by about $4 \mathrm{~min}$, and negative power density spikes of $\sim 2 \cdot 10^{-12} \mathrm{~W} / \mathrm{m}^{3}$, associated with velocity amplitudes of $v_{y}$ and $v_{z}$ of $30-60 \mathrm{~km} / \mathrm{s}$. These parameters, as well as the qualitative results are quite similar to those found by Marghitu et al. $(2005)^{1}$ and Hamrin et al. (2005) ${ }^{2}$. It is also noteworthy that the negative spikes of $\boldsymbol{j} \cdot \boldsymbol{E}$ coincide with negative spikes of $\partial\left(B^{2} / 2\right) / \partial t$ (Fig. 9c) of similar magnitude, so that both contribute about equally to a positive divergence of Poynting flux.

\section{Summary and conclusions}

Using resistive MHD simulations of magnetic reconnection in the magnetotail, we have investigated the release, transport, and conversion of energy in the tail. The dissipation in the MHD simulations was compared with the dissipation in a (more localized) particle simulation of reconnection. Here we summarize the major results:

(1) Energy release and conversion are not strongly localized, although the underlying instability is associated with a localized violation of MHD. As expected, the major energy release stems from the magnetic energy in the tail lobes, involving a major portion of the tail.

(2) Direct transfer from magnetic to thermal energy (Joule, ohmic dissipation) is unimportant for the overall energy balance, although it may be more relevant in a more localized simulation. Joule dissipation is localized similarly in particle and resistive MHD simulations. This result makes it plausible that the MHD results are applicable more generally even though the dissipation mechanism is probably not adequately described by a resistive term.

(3) Energy release from the lobes causes a divergence of Poynting flux, which is converted predominantly into

Vaivads, A.: Observations of concentrated generator regions in the nightside magnetosphere by Cluster/FAST conjunctions, Ann. Geophys., submitted, 2005. 


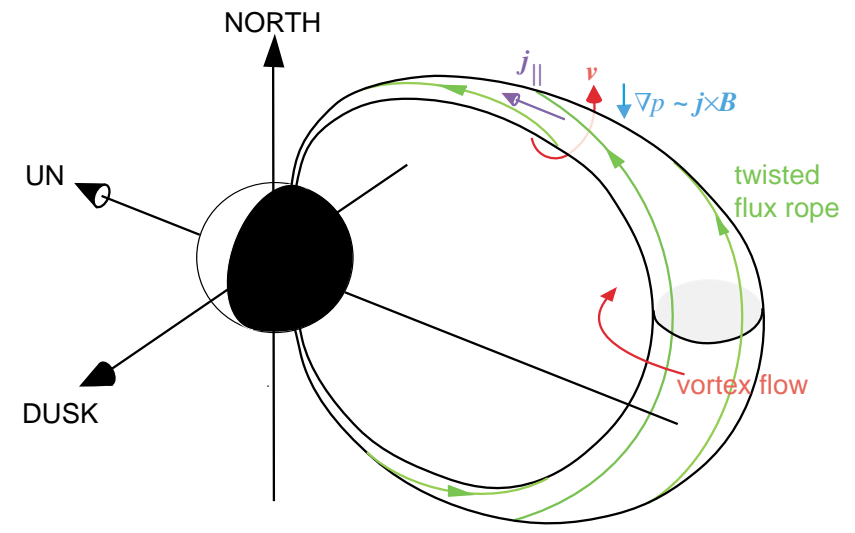

Fig. 10. Sketch of the generator mechanism in the near-Earth tail as inferred from the MHD simulation. The deflection of earthward flow leads to a build-up of field-aligned current inside a sheared or twisted magnetic flux tube. The associated upward (lobeward) flow is opposite to the direction of the strong pressure gradient and Lorentz force in the boundary regions of the plasma sheet, providing a generator with $\boldsymbol{j} \cdot \boldsymbol{E}<0$.

enthalpy flux at slow shock-like structures in the MHD simulation. Since there is no direct transfer from magnetic to thermal energy (or to the corresponding enthalpy flux), other than the Joule dissipation, kinetic energy acts as a mediator. Through approximate force balance a major part of the kinetic energy is transferred to thermal energy. Only some of the energy remains in kinetic energy flux. In contrast to the MHD simulations, our kinetic simulation does not show slow shocks. However, an equivalent transfer happens near the separatrices extending from the reconnection site.

(4) While the tailward transport, dominated by the enthalpy flux, is relatively unimpeded (apart from artificial boundary effects in the simulations), the earthward transport is slowed down and diverted when the flow reaches the dipolar region. It is noteworthy that the braking in the vicinity of the equatorial plane itself does not lead to significant local heating; more significant heating results from the slow-down of fieldaligned flow in the collapsing field at higher latitudes.

(5) From an auroral perspective it is of interest to investigate generator regions in the near tail. While the braking of the earthward flow near the equatorial plane is found to provide a generator term, as postulated by Shiokawa et al. (1997), this was found to have no strong near-Earth effect. More significant are generator regions off the equatorial plane, closely associated with vortical flow that twists flux tubes, causing field-aligned currents in the region 1 sense (that is, directed into the ionosphere on the dawn side and out of the ionosphere on the dusk side), as illustrated by Fig. 10. Although the flow plays a crucial role in this mechanism, the source of the energy that is turned into Poynting flux is not the kinetic energy but rather stems from a loss of enthalpy flux. This results in local heating and in mechanical work, which via approximate force balance between pressure gradient and Lorentz forces provides the energy transfer to magnetic energy.
The location of the generator regions in the plasma sheet boundary regions and the mechanism of outward motion in the direction of the pressure gradient force (and against the Lorentz force), as well as the estimated magnitudes of current density, cross-tail electric field, and negative $\boldsymbol{j} \cdot \boldsymbol{E}$ in our simulation are consistent with results from recent investigations of auroral generator regions with conjugated Cluster and FAST satellite data (Marghitu et al., 2005 ${ }^{1}$; Hamrin et al., 2005 ${ }^{2}$ ). While these observations were made both during growth phase and recovery of modest substorms, our simulation would most directly apply to the substorm expansion phase. It hence appears that the generator mechanism is not restricted to a particular substorm phase but, more universally, associated with fast earthward flows and their consequences.

Acknowledgements. This work was supported by the US Department of Energy's Office of Basic Energy Sciences through its Geosciences Research Program, by NASA's Sun Earth Connection Theory Program, and by the National Science Foundation through grant ATM-0202306.

Topical Editor T. Pulkkinen thanks S. W. H. Cowley and K. Rönnmark for their help in evaluating this paper.

\section{References}

Akhiezer, A. I., Akhiezer, I. A., Polovin, R. V., Sitenko, A. G., and Stepanov, K. N.: Plasma Electrodynamics, vol. 1: Linear Theory, Pergamon Press, New York, first English edn., 1975.

Birn, J. and Hesse, M.: Details of current disruption and diversion in simulations of magnetotail dynamics, J. Geophys. Res., 101, 15 345-15 358, 1996.

Birn, J., Hesse, M., Haerendel, G., Baumjohann, W., and Shiokawa, K.: Flow braking and the substorm current wedge, J. Geophys. Res., 104, 19895-19 904, 1999.

Hesse, M. and Winske, D.: Hybrid simulations of collisionless ion tearing, Geophys. Res. Lett., 20, 1207-1210, 1993.

Hesse, M., Schindler, K., Birn, J., and Kuznetsova, M.: The Diffusion Region in Collisionless Magnetic Reconnection, Phys. Plasmas, 6, 1781-1795, 1999.

Hesse, M., Birn, J., and Kuznetsova, M.: Collisionless magnetic reconnection: Electron processes and transport modeling, J. Geophys. Res., 106, 3721-3736, 2001.

Hesse, M., Kuznetsova., M., and Birn, J.: The role of electron heat flux in guide-field magnetic reconnection, Phys. Plasmas, 11, 5387-5397, 2004.

Lyons, L. R. and Pridmore-Brown, D. C.: Force balance near an X-line in a collisionless plasma, J. Geophys. Res., 95, $20903-$ $20909,1990$.

Shiokawa, K., Baumjohann, W., and Haerendel, G.: Braking of high-speed flows in the near-Earth tail, Geophys. Res. Lett., 24, 1179-1182, 1997.

Vasyliūnas, V. M.: Theoretical models of magnetic field line merging, Rev. Geophys. Space Phys., 13, 303-336, 1975.

Yin, L., Winske, D., Gary, S. P., and Birn, J.: Hybrid and Hall-magnetohydrodynamics simulations of collisionless reconnection: Effect of the ion pressure tensor and particle Hallmagnetohydrodynamics, Phys. Plasmas, 9, 2575-2584, 2002. 